\title{
Evaluation of an infant with hypercalcemia
}

\author{
Dganit Dinour • Liat Ganon
}

Received: 6 October 2014 / Revised: 6 October 2014 / Accepted: 14 October 2014 / Published online: 19 December 2014

(C) IPNA 2014

\section{Dear Editors,}

Guarino et al. recently published a clinical quiz in Pediatric Nephrology, titled "An infant with hypercalcemia" [1]. The quiz describes an infant with severe symptomatic hypercalcemia, nephrocalcinosis, and high vitamin $\mathrm{D}$ and low parathyroid hormone (PTH) serum levels. The authors present a list of causes of hypercalcemia in their Table 1 and ultimately conclude that the diagnosis in this case is vitamin $\mathrm{D}$ intoxication because the patient had been given a high dose of vitamin D supplement. However, they admit that this was previously common practice in their region-although they did not report any other cases of hypercalcemia. Indeed, treating infants with high vitamin $\mathrm{D}$ doses used to be a common practice in some regions, such as, for example, East Germany [2]. A very high and prolonged vitamin D intake may suffice to explain the clinical features of this patient, but the authors ignore recent publications suggesting that the most probable cause of hypercalcemia in infants is the entity previously referred to as "idiopathic infantile hypercalcemia." This entity has been recognized for many years, especially since vitamin D supplements became a common practice in the treatment of infants worldwide. In 2011, Schlingman et al. showed that infantile hypercalcemia develops in infants with homozygous or compound heterozygous loss-of-function mutations in the CYP24A1 gene which encodes 25-hydroxyvitamin D 24-hydroxylase, the enzyme responsible for degrading 1,25-dihydroxyvitamin $\mathrm{D}(1,25$ vitamin D) [2]. Loss-of-function mutations of this enzyme lead to the accumulation of 1,25 vitamin $\mathrm{D}$, hypercalcemia, hypercalciuria, nephrocalcinosis, and suppressed PTH, as in the case

D. Dinour $(\bowtie) \cdot$ L. Ganon

Department of Nephrology and Hypertension, Chaim Sheba Medical

Center, Tel-Hashomer, Israel

e-mail: dganit.dinour@sheba.health.gov.il

D. Dinour $\cdot$ L. Ganon

Sackler School of Medicine, Tel-Aviv University, Ramat Aviv, Tel Aviv, Israel presented by Guarino et al. [1]. Recently, CYP24A1 mutations have been shown by our group [3,4] and by others to cause not only neonatal hypercalcemia but also nephrocalcinosis and nephrolithiasis in children and adults. The adult patients may continue to suffer from hypercalcemia and kidney stones for many years and are at risk of chronic kidney failure. Although at this time no specific treatment is available, it is very important to diagnose patients with CYP24A1 mutations and to analyze their family members. Individuals carrying a CYP24A1 mutation should be advised to maintain an adequate water intake and a low sodium/low calcium diet, to avoid vitamin D supplement, a common practice nowadays also in adults, and avoid exposure to sunlight.

In conclusion, we strongly recommend that any patient with "idiopathic hypercalcemia" and/or unexplained nephrocalcinosis or kidney stones should be tested for 1,25 vitamin $\mathrm{D}$ levels and be genetically evaluated for the presence of a CYP24A1 mutation.

\section{References}

1. Guarino S, Marzuillo P, Apicella A, Annicchiarico Petruzzelli L, La Manna A (2014) An infant with hypercalcemia: questions. Pediatr Nephrol 29:2121

2. Schlingmann KP, Kaufmann M, Weber S, Irwin A, Goos C, John U, Misselwitz J, Klaus G, Kuwertz-Bröking E, Fehrenbach H, Wingen AM, Güran T, Hoenderop JG, Bindels RJ, Prosser DE, Jones G, Konrad M (2011) Mutations in CYP24A1 and idiopathic infantile hypercalcemia. N Engl J Med 365:410-421

3. Dinour D, Beckerman P, Ganon L, Tordjman K, Eisenstein Z, Holtzman EJ (2013) Loss-of-function mutations of CYP24A1, the vitamin D 24-hydroxylase gene, cause long-standing hypercalciuric nephrolithiasis and nephrocalcinosis. J Urol 190:552-557

4. Dinour D, Davidovits M, Aviner S, Ganon L, Michael L, ModanMoses D, Vered I, Bibi H, Frishberg Y, Holtzman EJ (2014) Maternal and infantile hypercalcemia aused by vitamin-Dhydroxylase mutations and vitamin D intake. Pediatr Nephrol 30: $145-152$ 